\title{
Heartbeat: Chronic inflammatory disorders and cardiovascular disease
}

\section{doi:10.1136/heartjnl-2016-310885}

Inflammation is thought to be a key pathophysiologic factor in atherosclerotic cardiovascular disease (CVD). In addition, patients with systemic inflammatory disorders, such as rheumatoid arthritis (RA), are at increased risk of CVD events, possibly modulated by disease-modifying anti-inflammatory therapy. ${ }^{1-3}$ However, few studies have examined whether these patients receive appropriate evaluation and treatment for conventional CVD risk factors in the primary care setting.

In this issue of Heart, Emanuel and colleagues (see page 1957) used electronic primary care health records to evaluate CVD risk factor measurement and treatment in $1121 \mathrm{RA}$ and 1875 inflammatory bowel disease (IBD) patients compared to control patients without these conditions. Evaluation of CVD risk factors was incomplete in most patients, with adequate data recorded for calculation of risk scores in only $11 \%$ of RA patients and 9\% of IBD patients (figure 1). On the other hand, prescription rates for antihypertensive medications in patients with RA were higher compared to controls over 5 years of follow-up (OR, 1.37, 95\% CI 1.14 to 1.65 ). A video abstract is also available for this article. http://heart.bmj.com/content/early/ 2016/09/07/heartjnl-2016-310111.full

In an editorial commenting on this study, González-Gay and GonzálezJuanatey (see page 1937) emphasize the suboptimal evaluation of CVD risk factors by both primary care providers and rheumatologists. They also emphasize the increased risk of CVD with the possibility that conventional risk scores may not provide adequate risk stratification for patients with systemic inflammatory disorders. Further, they suggest an algorithm for CVD risk stratification that incorporates additional markers of CVD risk, such as carotid ultrasound, to improve evaluation of this patient group (figure 2). They conclude: "Assessment of CVD risk must be routinely conducted in all the patients with chronic inflammatory diseases. Closer collaboration among clinicians is required to reduce the high rate of

Correspondence to Professor Catherine M Otto, Division of Cardiology, University of Washington, Seattle, WA 98195, USA; cmotto@u.washington.edu
CVD observed in patients with chronic inflammatory diseases."

Patients with diabetes are another group in whom conventional risk scores may underestimation the likelihood of adverse CVD events. ${ }^{4-6}$ In a prospective study of 746 patients with diabetes, Resl and colleagues (see page 1963) found that adding serum levels of growth differentiation factor 15 (GDF-15) and highsensitive troponin $\mathrm{T}$ (hs-TnT) to standard

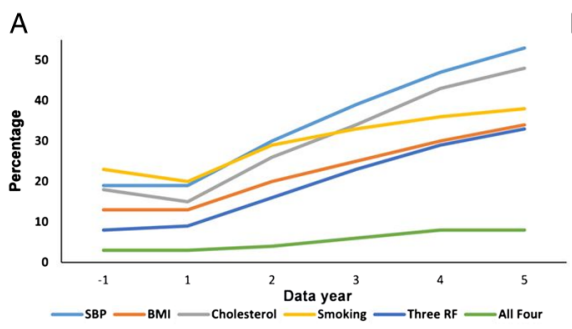

risk assessment that included $\mathrm{N}$-terminal pro B-type natriuretic peptide (NT-proBNP), resulted in net reclassification improvement of 33.6\% (figure 3).

Berezin puts this data in context in an editorial (see page 1939) that includes a table summarizing other biomarkers for CVD risk stratification in diabetic patients. He reminds us that "GDF-15 is closely associated with CVD including hypertrophy, heart failure, atherosclerosis,
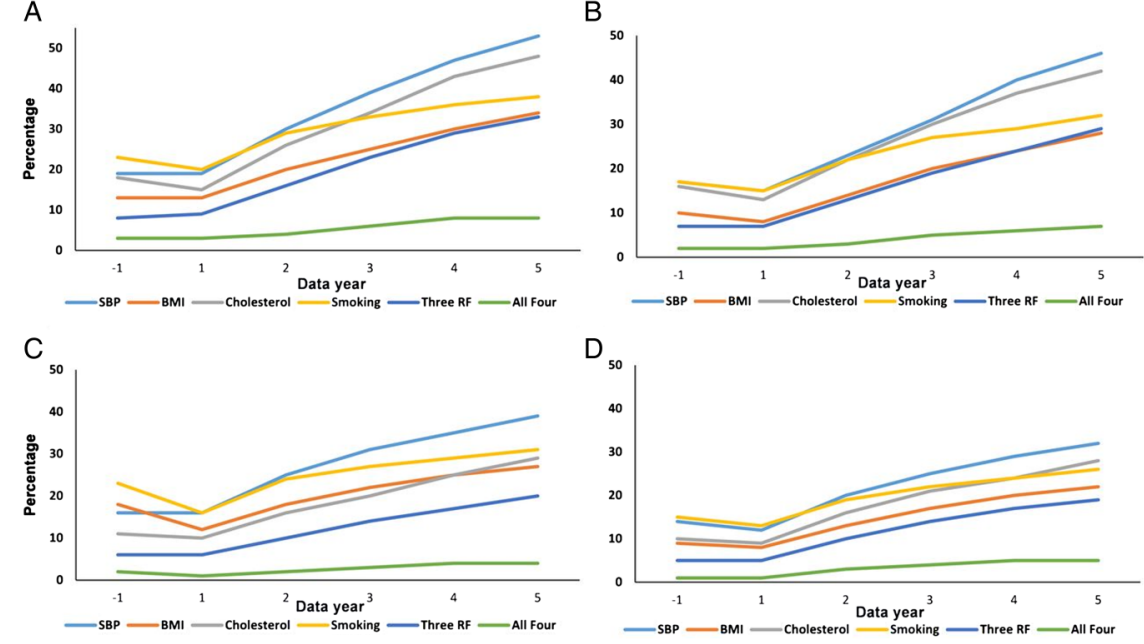

Figure 1 Cumulative trends in recording of vascular risk factors from the year before and up to 5 years following disease diagnosis in patients and controls. (A) Patients with rheumatoid arthritis. (B) Controls for rheumatoid arthritis. (C) Patients with inflammatory bowel disease. (D) Controls for inflammatory bowel disease. BMI, body mass index; RF, risk factors; SBP, systolic blood pressure.

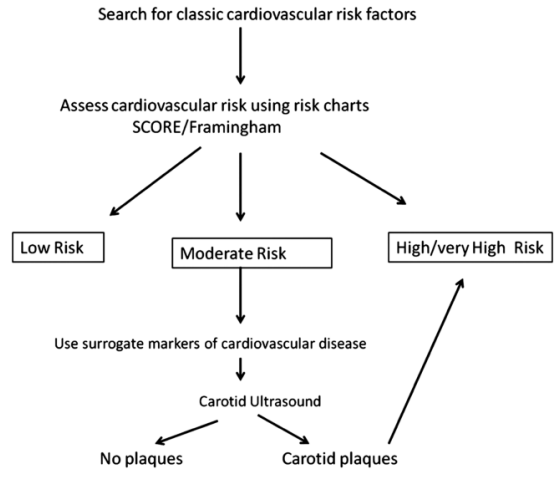

Figure 2 Proposed work-up to be used for the cardiovascular risk stratification in patients with chronic inflammatory diseases. SCORE, Systematic Coronary Risk Estimation.

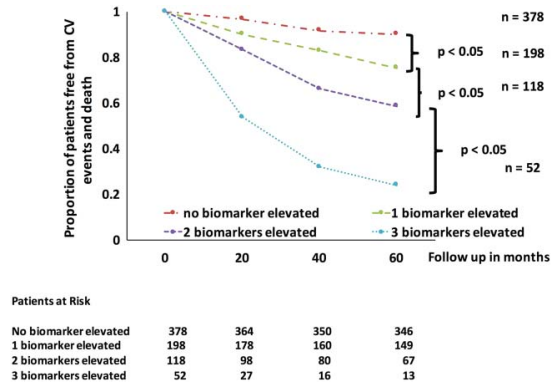

Figure 3 Kaplan-Meier plot complementary effects of GDF-15, hs-TnT and NT-proBNP. GDF-15, growth differentiation factor-15; hs-TnT, high-sensitive troponin T; NT-proBNP, $\mathrm{N}$-terminal pro B-type natriuretic peptide. 


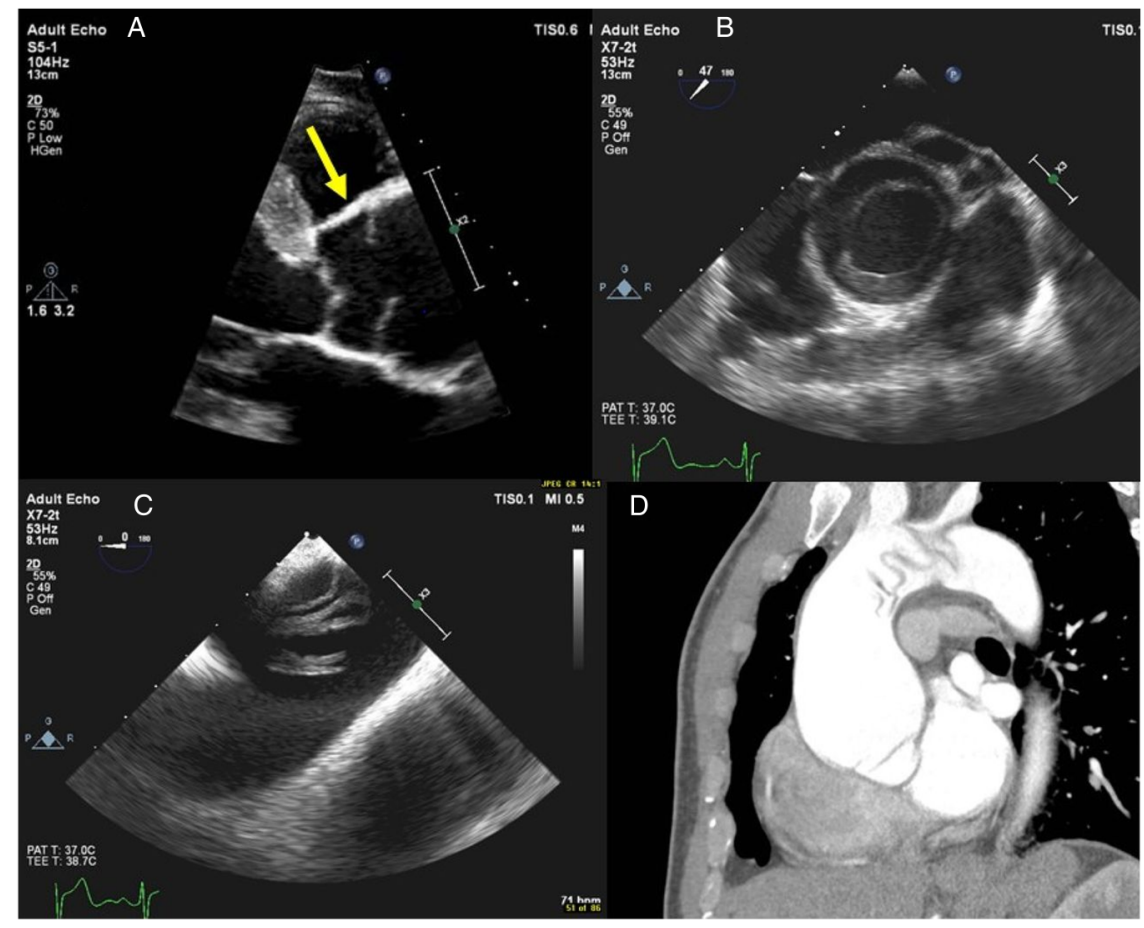

Figure 4 An unusual shadow above the aortic valve. What do you think it is?

endothelial dysfunction, as well as diabetes, obesity, insulin resistance and diabetes-related chronic kidney diseases." He also suggests: "Probably, other promising biomarkers such as galectin-3, lipoprotein-associated phospholipase A2, osteoprotegerin, endothelial progenitor cells and endothelial cell derived microparticles, which were reported closely in relation to CVD and death, might clear a situation around an individual prognostication in subjects with pre-diabetes and diabetes."

Antithrombotic therapy is the core element in prevention of embolic events in patients with atrial fibrillation (AF). ${ }^{7}$ However, some patients do not tolerate long term anticoagulation or have a contraindication due to bleeding risk or concurrent medical conditions. Because most thrombi associated with atrial fibrillation occur in the left atrial appendage (LAA), device closure of the LAA has been proposed as an approach to reduce thromboembolic risk in patients with nonvalvular AF, although this approach depends on the assumption that LAA thrombi are the only cause of ischemic stroke in AF patients. ${ }^{8}$

Berti and colleagues report a series of 110 patients with non-valvular AF. (see page 1969) The LAA occlusion device could be successfully placed without major complication in $96 \%$ of patients with a subsequent annual rate of ischemic stroke of $2.2 \%$ and a major bleeding rate of $1.1 \%$. The study group was similar to high risk AF patients seen in clinical practice - mean age $77 \pm 6$ years old $(62 \%$ men) with permanent AF in $64.5 \%$, persistent AF in $15.5 \%$ and paroxysmal AF in $20 \%$, of the study group. Mean $\mathrm{CHA}_{2} \mathrm{DS}_{2}$-VASc and HAS-BLED scores were $4.3 \pm 1.3$ and $3.4 \pm 1$, respectively.

The editorial by Swaans and Boersma (see page 1943) reviews the currently available LAA closure devices and concludes: "Further randomised trials with sufficient power to compare LAA closure to [direct oral anticoagulants or vitamin $\mathrm{K}$ antagonists] in patients at high risk of stroke and bleeding are urgently needed, especially in the case of the Amplatzer devices where no randomised trials are published in the present moment. With the currently available data, LAA closure in everyday practice outside a clinical trial may still only be used in those patients with $\mathrm{AF}$ and risk of stroke $\left(\mathrm{CHA}_{2} \mathrm{DS}_{2}\right.$-VASc score $\left.\geq 2\right)$ with a true contraindication to anticoagulation."

The Education in Heart article in this issue addresses genetic causes of dilated cardiomyopathy (DCM) (see page 2004). Although genetic testing now is widely available, diagnosis remains challenging due to the number of genes (over 100) reported to date and the variation in phenotypic presentation. Clinical screening of families is essential for either targeting genetic testing to specific genes (when suggested by family history and phenotype) or using a large panel of genes when a pre-test likely diagnosis is not evident. Identification of a genetic cause for DCM allows tailored clinical monitoring and medical treatment for all family members.

The Image Challenge in this issue (see page 1942) shows an unusual finding on echocardiographic and CT imaging. The online videos and discussion are illuminating (figure 4).

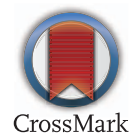

To cite Otto CM. Heart 2016;102:1935-1936.

Heart 2016;102:1935-1936.

doi:10.1136/heartjnl-2016-310885

\section{REFERENCES}

1 Weiner SD, Ahmed HN, Jin Z, Cushman M, Herrington DM, Nelson JC, Di Tullio MR, Homma S. Systemic inflammation and brachial artery endothelial function in the Multi-Ethnic Study of Atherosclerosis (MESA). Heart. 2014;100:862-6.

2 Agca R, Heslinga SC, van Halm VP, Nurmohamed MT. Atherosclerotic cardiovascular disease in patients with chronic inflammatory joint disorders. Heart 2016;102:790-5.

3 Sintek MA, Sparrow CT, Mikuls TR, Lindley KJ, Bach RG, Kurz HI, Novak E, Singh J. Repeat revascularisation outcomes after percutaneous coronary intervention in patients with rheumatoid arthritis. Heart. 2016;102:363-9.

4 Johansson I, Dahlström U, Edner M, Näsman P, Rydén L, Norhammar A. Risk factors, treatment and prognosis in men and women with heart failure with and without diabetes. Heart. 2015;101: $1139-48$

5 van der Leeuw J, van Dieren S, Beulens JW, Boeing $H_{\text {, }}$ Spijkerman AM, van der Graaf $Y$, van der A DL, Nöthlings U, Visseren FL, Rutten GE, Moons KG, van der Schouw YT, Peelen LM. The validation of cardiovascular risk scores for patients with type 2 diabetes mellitus. Heart. 2015;101:222-9.

6 Brownrigg JR, de Lusignan S, McGovern A, Hughes C, Thompson MM, Ray KK, Hinchliffe RJ. Peripheral neuropathy and the risk of cardiovascular events in type 2 diabetes mellitus. Heart. 2014;100:1837-43.

7 Senoo K, Lip GY. Prediction of stroke in patients without atrial fibrillation using the CHADS2 and CHA2DS2-VASC scores: a justification for more widespread thromboprophylaxis? Heart. 2014;100:1485-6.

8 Akoum N. New perspectives on atrial fibrillation and stroke. Heart. 2016 Aug 24. pii: heartjnl-2015-309066 